\title{
Prevalence of Enchondromas of the Proximal Femur in Adults as an Incidental Finding on MRI of the Pelvis
}
A. M. Davies ${ }^{1}$
A. Patel ${ }^{1}$
C. Azzopardi ${ }^{1}$
S. L. James ${ }^{1}$
R. Botchu ${ }^{1}$
${ }^{1}$ Department of Musculoskeletal Radiology, The Royal Orthopaedic
Address for correspondence Rajesh Botchu, FRCR, Department of Hospital, Birmingham, United Kingdom Musculoskeletal Radiology, The Royal Orthopedic Hospital, Bristol Road South, Northfield, Birmingham B31 2AP, United Kingdom Indian J Radiol Imaging 2021;31:582-585. (e-mail: drbrajesh@yahoo.com).

\begin{abstract}
Objective To determine the prevalence of enchondroma in adults as an incidental finding in the proximal femur on magnetic resonance imaging (MRI).

Materials and Methods A retrospective review of the MRI scans of the pelvis in a series of adult patients was conducted. All presented with nononcological musculoskeletal complaints. The site, size, and appearances of the enchondromas were identified according to criteria from previous studies.

Results A total of 1,209 proximal femora in 610 patients were reviewed and a total of 9 enchondromas were identified. These ranged from 0.6 to $2.5 \mathrm{~cm}$ in length (mean $1.3 \mathrm{~cm}$ ). None showed aggressive features suggestive of malignancy. Three cases (33\%)

\section{Keywords}

- enchondroma

- proximal femur

- MRI underwent follow-up MRI scans which showed no change in size or morphology. Conclusion The prevalence on MRI of incidental enchondromas arising in the proximal femur is $0.7 \%$. This is three to four times less common than seen in the proximal humerus and around the knee.
\end{abstract}

\section{Introduction}

Central cartilage tumors (CCTs) are neoplasms of the bony medulla composed of hyaline cartilage. They range from the benign enchondroma, the second commonest bone tumor after osteochondroma, through ascending grades of chondrosarcoma reflecting increasing malignancy. ${ }^{1}$ Enchondromas are a common incidental finding in the short and long tubular bones on magnetic resonance imaging (MRI), particularly around the knee ${ }^{2}$ and in the proximal humerus. ${ }^{3}$ With the increasing use of MRI in the investigation of musculoskeletal (MSK) disorders, it is useful to know the prevalence of incidental enchondromas at different sites to help distinguish them from other possibly malignant entities. Four previous studies have addressed this issue. Three looked at knee MRI scans and identified incidental enchondromas on 0.8 to $2.9 \%$ routine studies. ${ }^{4-6}$ The fourth found incidental enchondromas in the proximal humerus on $2.1 \%$ of routine MRI of the shoulder studies. ${ }^{7}$ The purpose of this retrospective study was to establish the prevalence of enchondromas of the proximal femur on routine MRI scans of the pelvis as an aid to the differential diagnosis of bone lesions at this particular site.

\section{Materials and Methods}

A series of patients, all postskeletal fusion, who had undergone MRI of the pelvis for various MSK complaints were identified from the picture archiving communication system of a specialist orthopaedic hospital. All referrals originating
DOI https://doi.org/ $10.1055 / \mathrm{s}-0041-1735915$. ISSN $0971-3026$. (c) 2021. Indian Radiological Association. All rights reserved.

This is an open access article published by Thieme under the terms of the Creative Commons Attribution-NonDerivative-NonCommercial-License, permitting copying and reproduction so long as the original work is given appropriate credit. Contents may not be used for commercial purposes, or adapted, remixed, transformed or built upon. (https://creativecommons.org/ licenses/by-nc-nd/4.0/)

Thieme Medical and Scientific Publishers Pvt. Ltd., A-12, 2nd Floor, Sector 2, Noida-201301 UP, India 
from an orthopaedic oncology source were excluded as was any case where previous imaging had revealed an enchondroma or where the clinical details supplied could be attributed to an enchondroma. Three experienced MSK radiologists independently retrospectively reviewed onethird of the scans of 610 patients to identify the presence or absence of enchondromas of the proximal femur. In cases where there was any doubt, the decision was made by consensus of two of the radiologists. No attempt was made to assess other bone lesions identified in the proximal femur in this series. Local committee approval was obtained for this study as part of a service evaluation.

All MRI examinations were performed in a single institution on a 1.5 or 3T strength scanner (1.5T and 3T Siemens, Erlangen, Germany). Minimum protocol for each examination comprised coronal and axial T1-weighted fast spin-echo, coronal short-tau inversion recovery (STIR), and axial T2weighted fat-suppressed fast spin-echo sequences with a relatively large field of view (FOV) to include the whole pelvis and proximal third of the femora. Isolated dedicated scans utilizing a small FOV (e.g., young adult hip or groin pain protocols) were excluded from the series as it could not be certain that localized symptoms could not be due to the enchondroma. The diagnosis of enchondroma was established according to the criteria of a previous study relating to enchondromas identified on knee MRI scans. ${ }^{4}$ These comprised a nonsubchondral, intramedullary mass with a lobular outline relatively hypointense on the T1-weighted and markedly hyperintense on the T2-weighted and STIR images.

Cases with an enchondroma identified were further evaluated for site (femoral head, neck, or proximal diaphysis), size, and presence/absence of endosteal scalloping. Patient demographics such as age and sex were compared with the total patient population. Any change in the size of the enchondroma was recorded in those cases that had undergone follow-up studies for whatever reason.

\section{Results}

The sex and age ranges are shown by decade in $\mathbf{- F i g . ~} \mathbf{1}$ for all 619 patients; $27 \%$ of patients were male and $73 \%$ were female. A unilateral hip prosthesis was found in 11 cases

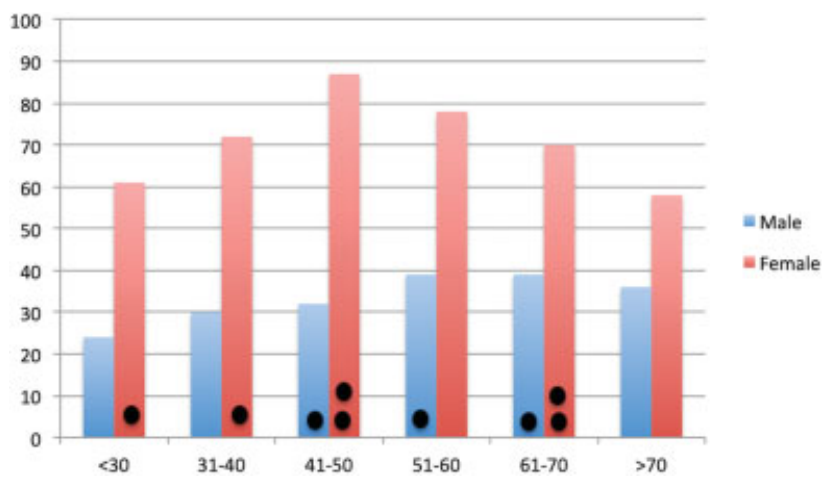

Fig. 1 Number of patients by age and sex in decades. The nine incidental enchondromas are shown as the black circles over the relevant age and sex categories.

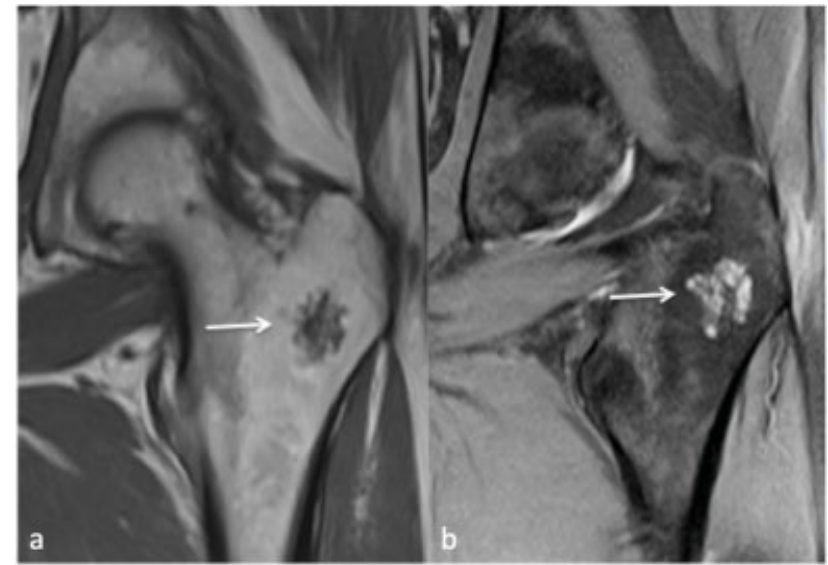

Fig. 2 (a) Coronal T1-weighted and (b) STIR images show a 2.3-cm intertrochanteric enchondroma (arrow). Note the typical hypointense on T1 and hyperintense on STIR lobulated cartilaginous contour and internal structure. STIR, short-tau inversion recovery.

leaving a total study series of 1,209 proximal femora. Nine enchondromas were found yielding a prevalence of incidental enchondromas as $1.4 \%$ on routine pelvic MRI examinations which equate to $0.7 \%$ of the proximal femora.

In the enchondroma group, six (66\%) patients were female and three (33\%) were male. Patients' age ranged from 23 to 65 years (mean 48 years) (-Fig. 1). Eight (89\%) arose in the intertrochanteric portion of the proximal femur (metaphyseal equivalent) with one in the head (epiphyseal equivalent). Five were eccentric and four were central in location. The maximum length of the lesions ranged from 0.6 to $2.5 \mathrm{~cm}$ (mean $1.3 \mathrm{~cm}$ ) (-Fig. 2). Three cases underwent follow-up MRI scans 7 months, 51 months, and 5 years later for unrelated clinical reasons with none showing any change in size or morphology when comparing the scans (-Fig. 3). None of the cases showed any aggressive features (e.g., endosteal scalloping or cortical destruction) that might be construed as indicative of malignant transformation.

\section{Discussion}

To the best of our knowledge, this is the first study to determine the incidental prevalence on MRI of enchondroma in the proximal femur. At $0.7 \%$, the prevalence is four times less common than most commonly quoted articles on the knee $^{4,6}$ and three times less common than in the proximal humerus. ${ }^{7}$ It is, therefore, a relatively exceptional incidental finding particularly if one takes into account the significantly greater numbers of knee and shoulder MRI scans performed routinely in most units.

Typically, the enchondroma arises in the intertrochanteric portion of the proximal femur with a mean length of $1.3 \mathrm{~cm}$ (-Figs. 2 and 3 ). This study shows a female:male preponderance of $2: 1$ for enchondromas that is a similar finding to other relatively recent publications on CCTs of long bones. ${ }^{8-11}$ However, when taking into account the sex distribution in the current series ( $\mathbf{- F i g . 1} \mathbf{1}$ ), the true sex ratio in this study for incidental enchondroma is approximately even. 


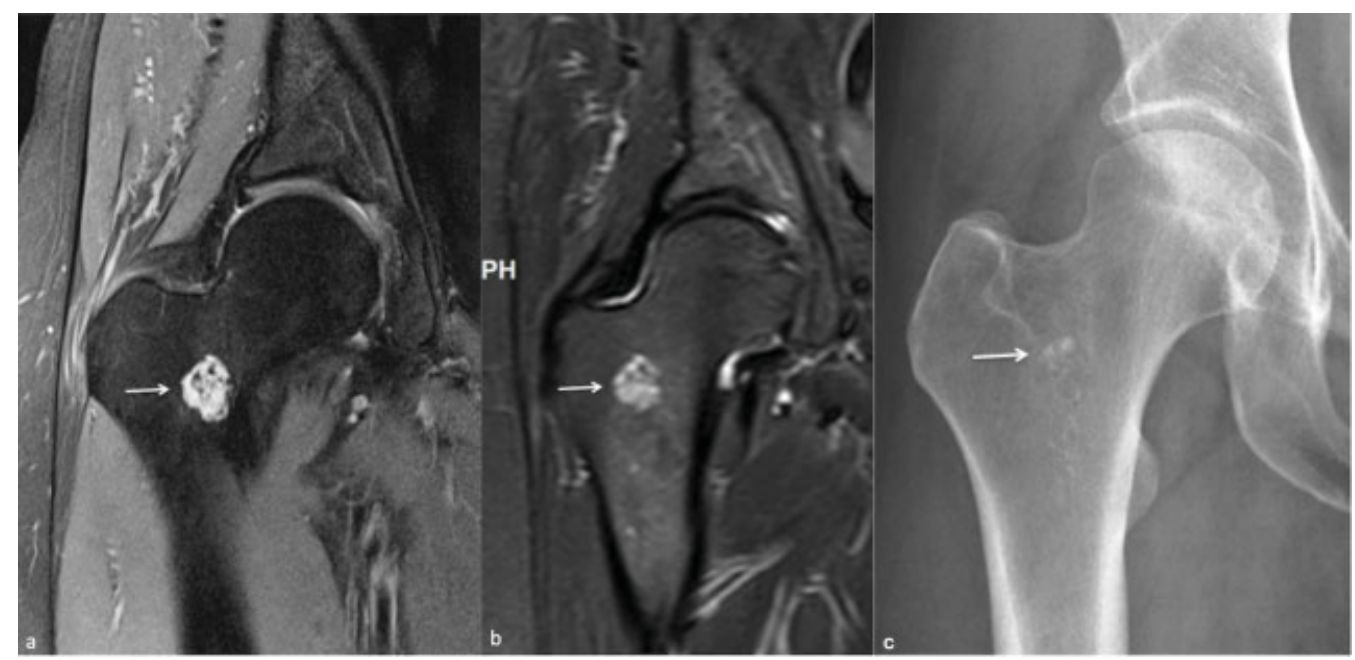

Fig. 3 (a) Coronal fat-suppressed proton density image showing a 1.9-cm intertrochanteric enchondroma (arrow). (b) Coronal STIR image obtained 5 years later shows no significant change in size or morphology. The small foci of signal void corresponds to the subtle chondroid calcification on the (c) AP radiograph of the hip (arrow). AP, anteroposterior; STIR, short-tau inversion recovery.

The differential diagnosis of benign femoral neck lesions depends on patient's age and site. Osteoid osteomas tend to occur in the young, typically involving the inferior cortex. Fibrocystic change in the anterolateral femoral neck can be associated with femoroacetabular impingement frequently identified in young adults, whereas solitary foci of fibrous dysplasia and bone islands may be identified at any age. It is beyond the scope of this article to describe their particular imaging features suffice to say that there is usually little confusion with CCTs in the proximal femur.

The distinction of enchondroma from the borderline malignancy arising in the appendicular skeleton, the atypical cartilaginous tumor formerly known as grade 1 or low-grade central chondrosarcoma, ${ }^{12}$ is problematic both on imaging and pathology. ${ }^{8,13-16}$ Recent studies have highlighted the increased identification of CCTs attributed to the ever-increasing use of MRI particularly in the proximal humerus and around the knee. ${ }^{17,18}$ Guidelines and imaging protocols for the management and follow-up have been proposed in an attempt to reduce costly and in many instances diagnostically low-yield follow-up imaging studies. ${ }^{9}{ }^{11,19-21}$ Whether the enchondromas retrospectively identified in this study merited any follow-up is debatable as the three cases (33\%) that did undergo a repeat MRI scan showed no change including one after 5 years (-Fig. 3). Also, none showed any of the imaging features that might have been a cause of concern, that is, all were less than $5 \mathrm{~cm}$ in length with absence of endosteal scalloping and cortical remodeling/thickening (- Fig. 2). ${ }^{22}$

Limitations of this study include the retrospective design with lack of uniformity of all the scan parameters. Also, although the criteria set for the diagnosis of enchondroma are widely accepted, there is no definitive histological confirmation in this series. In addition, there is some patient selection bias in that the clinical indications for the scans were varying MSK complaints referred from primary and secondary care excluding orthopaedic oncology. Was one to select patients undergoing MRI of the pelvis including the proximal femora for non-MSK complaints (e.g., gyneco- logical and prostate scans) then it might be anticipated that the prevalence of incidental enchondromas could be similar or even less than the $0.7 \%$ identified in this study.

\section{Conclusion}

This study establishes that the prevalence on MRI of incidental enchondromas arising in the proximal femur to be $0.7 \%$ which is three to four times less common than seen in the proximal humerus and around the knee. Radiologists should be aware of the prevalence of this entity when venturing a differential diagnosis for bone lesions at this anatomical site.

\section{Disclosure}

No financial disclosure.

Conflict of Interest

None declared.

\section{References}

1 Suster D, Hung YP, Nielsen GP. Differential diagnosis of cartilaginous lesions of bone. Arch Pathol Lab Med 2020;144(01):71-82

2 Kransdorf MJ, Peterson JJ, Bancroft LW. MR imaging of the knee: incidental osseous lesions. Magn Reson Imaging Clin N Am 2007; 15(01):13-24

3 Douis H, Saifuddin A. The imaging of cartilaginous bone tumours. I. Benign lesions. Skeletal Radiol 2012;41(10):1195-1212

4 Walden MJ, Murphey MD, Vidal JA. Incidental enchondromas of the knee. AJR Am J Roentgenol 2008;190(06):1611-1615

5 Grainger R, Stuckey S, O'Sullivan R, Davis SR, Ebeling PR, Wluka AE. What is the clinical and ethical importance of incidental abnormalities found by knee MRI? Arthritis Res Ther 2008;10 (01):R18

6 Stomp W, Reijnierse M, Kloppenburg M, et al; NEO study group. Prevalence of cartilaginous tumours as an incidental finding on MRI of the knee. Eur Radiol 2015;25(12):3480-3487

7 Hong ED, Carrino JA, Weber KL, Fayad LM. Prevalence of shoulder enchondromas on routine MR imaging. Clin Imaging 2011;35 (05):378-384 
8 Crim J, Schmidt R, Layfield L, Hanrahan C, Manaster BJ. Can imaging criteria distinguish enchondroma from grade 1 chondrosarcoma? Eur J Radiol 2015;84(11):2222-2230

9 Sampath Kumar V, Tyrrell PNM, Singh J, Gregory J, Cribb GL, Cool P. Surveillance of intramedullary cartilage tumours in long bones. Bone Joint J 2016;98-B(11):1542-1547

10 Deckers C, Schreuder BHW, Hannink G, de Rooy JW, van der Geest IC. Radiologic follow-up of untreated enchondroma and atypical cartilaginous tumors in the long bones. J Surg Oncol 2016;114 (08):987-991

11 Davies AM, Patel A, James SL, Azzopardi C, Botchu R. An imaging protocol for the management of central cartilage tumours of the proximal fibula. Clin Radiol 2020;75(09):714.e1-714.e6

12 Bovée JVMG, Bloem JL, Flanagan AM, et al. Central atypical cartilaginous tumour/chondrosarcoma, grade 1. In: WHO Classification of Tumours. Soft Tissue and Bone Tumours. 5th ed. Lyon: WHO Classification of Tumours Editorial Board International Agency for Research on Cancer; 2020:370-372

13 Skeletal Lesions Interobserver Correlation among Expert Diagnosticians (SLICED) Study Group. Reliability of histopathologic and radiologic grading of cartilaginous neoplasms in long bones. J Bone Joint Surg Am 2007;89(10):2113-2123

14 Eefting D, Schrage YM, Geirnaerdt MJ, et al; EuroBoNeT consortium. Assessment of interobserver variability and histologic parameters to improve reliability in classification and grading of central cartilaginous tumors. Am J Surg Pathol 2009;33(01): 50-57

15 Afonso PD, Isaac A, Villagrán JM. Chondroid tumors as incidental findings and differential diagnosis between enchondromas and low-grade chondrosarcomas. Semin Musculoskelet Radiol 2019; 23(01):3-18

16 van de Sande MAJ, van der Wal RJP, Navas Cañete A, et al. Radiologic differentiation of enchondromas, atypical cartilaginous tumors, and high-grade chondrosarcomas-Improving tumor-specific treatment: a paradigm in transit? Cancer 2019;125 (19):3288-3291

17 van Praag Veroniek VM, Rueten-Budde AJ, Ho V, Dijkstra PDS, Fiocco M, van de Sande MAJStudy group Bone and Soft tissue tumours (WeBot) Incidence, outcomes and prognostic factors during 25 years of treatment of chondrosarcomas. Surg Oncol 2018;27(03):402-408

18 Patel A, Davies AM, Botchu R, James S. A pragmatic approach to the imaging and follow-up of solitary central cartilage tumours of the proximal humerus and knee. Clin Radiol 2019;74(07):517-526

19 Parlier-Cuau C, Bousson V, Ogilvie CM, Lackman RD, Laredo JD. When should we biopsy a solitary central cartilaginous tumor of long bones? Literature review and management proposal. Eur J Radiol 2011;77(01):6-12

20 Davies AM, Patel A, James SL, Botchu R. A retrospective validation of an imaging protocol for the management of solitary central cartilage tumours of the proximal humerus and around the knee. Clin Radiol 2019;74(12):962-971

21 Jurik AG, Hansen BH, Weber K. Solitary enchondromas-diagnosis and surveillance: Danish guidelines. Radiologe 2020;60(Suppl 1): 26-32

22 Murphey MD, Flemming DJ, Boyea SR, Bojescul JA, Sweet DE, Temple HT. Enchondroma versus chondrosarcoma in the appendicular skeleton: differentiating features. Radiographics 1998;18 (05):1213-1237, quiz 1244-1245 